\title{
Clinically Assessing Remineralizing Therapies using Quantitative Light- induced Fluorescence: A Pilot Study
}

\section{R. Vaderhobli ${ }^{1 *}$, L. Saunders ${ }^{2}$, R. Billings ${ }^{2}$, C. Feng ${ }^{2}$ and H. Malmstrom ${ }^{2}$}

${ }^{1}$ Department of Preventive and Restorative Dentistry, UCSF School of Dentistry, San Francisco, USA

${ }^{2}$ Eastman Dental Center at University of Rochester, Rochester, USA

\begin{abstract}
Quantitative light-induced fluorescence (QLF) method has been used in vitro, in situ and in vivo as a diagnostic method in assessing smooth surface caries over the last twenty years. Coincidentally, reversal or remineralization of early smooth surface lesion by remineralizing agents has been substantiated by in vitro and in situ studies over the last twenty years. However, there are very few reported clinical trials to assess the effectiveness of these remineralizing agents by using QLF. This research aims at exploring the remineralizing potential of a specially formulated remineralizing mouth rinse and the 'gold-standard' Sodium Fluoride (NaF) rinse by using QLF as a monitoring tool.

Methods: Twelve Subjects at high-risk for caries progression with smooth surface caries on anterior teeth were selected and randomly assigned to the control and to the test group. Twice daily (after breakfast and after dinner) the subjects rinsed $1 \mathrm{oz}$ of either the test (Calcium and Fluoride; $250 \mathrm{ppm} \mathrm{F}$ ) or the control rinse (NaF; $250 \mathrm{ppm} \mathrm{F}$ ). Baseline assessments of the progression of the smooth surface lesion were done by QLF at $0,1,2$, and end of 3 months. A two-sided t-test, MANOVA and exact F-statistics were used to determine differences between treatment groups.
\end{abstract}

Results: There was no significant difference between the two groups in preventing the progression of caries ( $p>$ 0.05). It was concluded that QLF could be a useful diagnostic tool to aid the clinician in caries management.

\section{Introduction}

The consensus development conference on the Diagnosis and Management of Dental Caries Throughout Life, sponsored by the National Institutes of Health, 2001 concluded that "improved diagnosis of early non-cavitated lesions and treatment for prevention and arrest of such lesions" should form the basis for managing dental caries by the practicing dentists [1]. In a recent US survey, dental caries still continues to be a major disease affecting adults ( 18 years and older) with $94 \%$ of them showing evidence of treated or untreated coronal caries [2]. The standard procedure employed by any Dentist in mitigating a high risk for caries patient has always been the brush, floss, prescription of fluoridated toothpastes, fluoride rinses, remineralizing rinses. These standard procedures have shown to be effective in reducing the risk for some population at lower risk but these measures do not target the population for greatest risk to develop caries. The caries diagnostic tool most commonly employed by the dentist includes visual examination, explorer, mirror and radiography. The shortcoming is that these methods are unable to detect the carious lesions at an earlier stage and assign them into various possible risk categories. To mitigate these issues both for research purposes and for clinical practice a very sensitive and clinically applicable method for early detection and quantification of caries lesion namely quantitative light-induced fluorescence (QLF, Inspektor Research Systems, Amsterdam, The Netherlands) was developed [3-8].

QLF device uses a halogen light within the visible frequency to scan the entire tooth surface. This surface can then be captured by a microCCD-video camera and stored in the in-built computer for future analysis. The analytical software aids the analyst to define lines around the selected lesion of interest. Then the software simulates sound enamel in the actual region of demineralization by using fluorescent radiance along the line of the lesion. The loss of fluorescent intensity in the lesion is calculated by the difference in fluorescent radiance between the simulated sound area and the actual carious area. This is expressed as a percentage of that of sound enamel [9]. QLF has become popular over other caries diagnostic aid mainly because of its correlation with mineral changes and micro radiography/histology and for in vitro repeatability [10-13].

The equilibrium between protective and pathological factors determines the caries progression (demineralization) or reversal (remineralization) and the key agent responsible for battling caries appears to be fluoride [14]. However, due to lack of adequate statistical testing and limited number of studies based on a evidence based systematic review it was concluded that use of fluoride may be insufficient for reversing progression of early caries lesions [15]. Consequently, various remineralization agents like calcium and phosphates (to name a few) were developed which were combined with fluoride to enhance fluoride's anti caries effectiveness [16]. Application of QLF device in clinical trials to assess the remineralization of a carious lesion by remineralizing therapy is still lacking in numbers.

The aim of this study was to test clinically if QLF would be a suitable tool to monitor the progress of lesions in high risk for caries progression population who were rinsing either with a fluoride or a calcium remineralizing solution. The null hypothesis to be tested was that there would be no difference among the two treatment groups.

*Corresponding author: R. Vaderhobli, Department of Preventive and Restorative Dentistry, UCSF School of Dentistry, 707 Parnassus Avenue, Room D 1028, San Francisco, CA 94143-0758, USA, Tel: 415476 4469; Fax: 415476 0858; E-mail: Ram.Vaderhobli@ucsf.edu

Received November 12, 2011; Accepted December 22, 2011; Published January 07, 2012

Citation: Vaderhobli R, Saunders L, Billings R, Feng C, Malmstrom H (2012) Clinically Assessing Remineralizing Therapies using Quantitative Light-induced Fluorescence: A Pilot Study. Dentistry 2:112. doi:10.4172/2161-1122.1000112

Copyright: (c) 2012 Vaderhobli R, et al. This is an open-access article distributed under the terms of the Creative Commons Attribution License, which permits unrestricted use, distribution, and reproduction in any medium, provided the original author and source are credited. 


\section{Materials and Methods}

The experimental design of the pilot clinical trial is schematically outlined in Figure 1. This double-blinded, randomized pilot clinical trial was carried out according to National Institute of Health Policies on conducting clinical trials with human participants [17] in Monroe Community Hospital (MCH, Rochester, NY). Prior to the start of the study the protocol was reviewed and approved by the appropriate institutional review board at the University of Rochester (Rochester, $\mathrm{NY}$ ) and $\mathrm{MCH}$. The inclusion criteria were the ability to sign informed consent, presence of minimum five to ten natural uncrowned teeth, presence of at least one to two smooth surface caries and no history of allergy to any ingredients relevant to the test products as determined by the dental professional monitoring the study. Exclusion criteria were gross dental neglect, greater than grade 2 or more mobility of teeth, presence of extensive cavitated smooth surface lesion and subjects who receive dental treatment during this study. Twenty-six subjects (> 60 yrs) were screened using the QLF and visual assessment.

The QLF system consisted of a portable intra-oral camera device connected to a computer fitted with a frame grabber. Baseline examination (July-August, 2006) was done on anterior teeth with caries

Approval by RSRB committee from both Univ. of Rochester and Monroe Community hospital

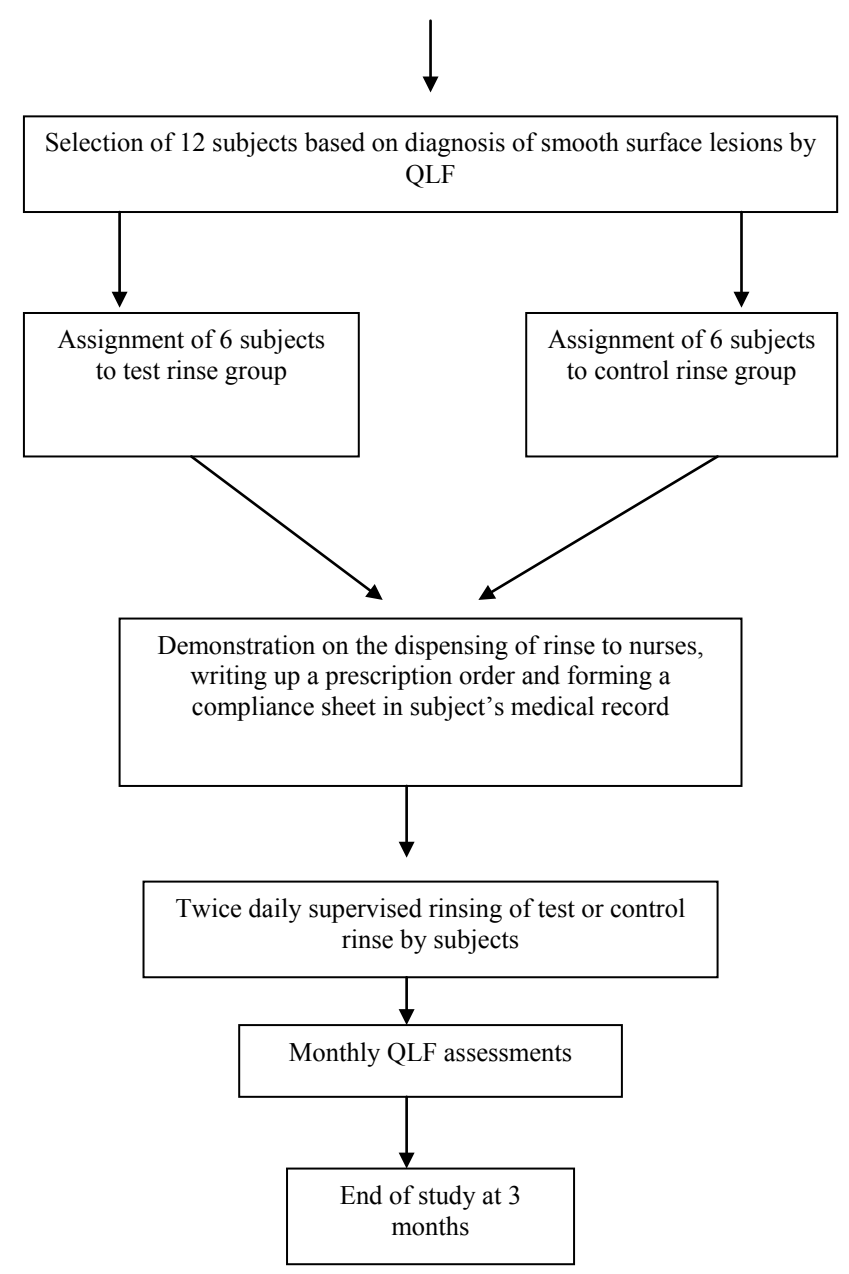

Figure 1: Experimental design of the clinical trial lesions by shining the blue light from the light source $\left(\lambda_{\max }=370 \mathrm{~nm}\right.$, full spectral width at Half the Maximal Value $=80 \mathrm{~nm}$ ). The fluorescent images of the tooth were captured by a micro-CCD video camera with a signal-to-noise ratio of $44 \mathrm{~dB}$ and equipped with a high pass-filter (orange, $\lambda>540 \mathrm{~nm}$ for the laser light). The captured images of the lesions were digitized by the frame grabber and were quantitatively analyzed with the QLF software. The principal investigator who also was the image analyst selected the images of lesions with incipient caries or demineralization and 12 subjects were chosen to participate based on the diagnosis of the smooth surface lesions and were randomly distributed into two groups (control and experimental) of six each as generated by the computer.

Both the experimental and the control rinse were prepared at the American Dental Association-Paffenbarger Research Center at the National Institute of Standards and Technology (ADA-PRC, Gaithersburg, MD). The experimental rinse contained $21.9 \mathrm{mmol} / \mathrm{L}$ $\mathrm{Ca}$ (solution 1, yellow) and 26.3-mmol/L fluoride (F) from $\mathrm{NaF}$ and $2-\mathrm{mmol} / \mathrm{L}$ citric acid (solution 2, red). Control rinse contained 13.2- $\mathrm{mmol} / \mathrm{L} \mathrm{F}$ from $\mathrm{NaF}$ (solution 3, blue) and 13.2-mmol/L F from $\mathrm{NaF}$ (solution 4 , green). When equal volumes of solution 1 and 2 or 3 and 4 were mixed the rinse contained $250 \mathrm{ppm}$ of $\mathrm{F}$ and had to be used immediately to achieve anticipated efficacy. The rinse solutions with the color codes were stored in $10 \mathrm{oz}$ partitioned bottles and were marked accordingly for either the control or the experimental rinsing group and the bottles had similar appearance to ensure blindness of the trial. Nurse Managers and nurses from each ward where the subjects resided were given a demonstration on the correct manner of dispensing the rinse and were instructed to check off the compliance sheet daily after administering the rinse once in the morning after breakfast and once in the night after dinner. No oral prophylaxsis were carried out prior to the start of the study. The nurses in-charge dispensed $10 \mathrm{oz}$ of either the control or the test rinse in a small cup and ensured the subjects rinsed for one minute after breakfast and dinner during the three month period. The subjects were asked not to eat, drink or brush for 30 minutes after rinsing. Each subject had two bottles of either the experimental or the control rinse. The principal investigator, subjects and nurses in charge of the subjects were blinded as to the group assignment of the rinses.

Examinations to monitor the mineral changes in these incipient lesions were done at the end of first month (September, 2006), end of second month (October, 2006) and after the end of third month (November, 2006) by positioning and shining the light guide perpendicular to the tooth. A standard hydration condition was maintained by asking the subjects to wet and wipe the teeth with their saliva and tongue. Three images each of the natural incipient white spot lesions were captured. Capturing of a good image took approximately 10 seconds and only one master lesion was considered in this study. The QLF calibrated principal investigator completed all the examinations under room light and the images were then stored for future analysis in the computer program (Inspektor Research Systems BV, Amsterdam, The Netherlands). Image analysis was done using the on-screen guidance which consisted of selecting reference points and an individual patch was created for the lesion as shown in Figures 2 $\mathrm{A}$ and $2 \mathrm{~B}$. The software program scaled the new images to the earlier baseline image and then calculated the lesion size as white spot area (WSA) in $\mathrm{mm}^{2}$, fluorescence loss as $\Delta \mathrm{F}$, and fluorescence loss integrated over the lesion as $\Delta \mathrm{Q}$ (fluorescence loss $\mathrm{x} \mathrm{mm}^{2}$ ) [18] as shown in Figure 2 C. Only successful reconstruction pattern were accepted. On every examination an accuracy of $95 \%$ was set to capture images and the created individual patch for each lesion was superimposed to all images (second and third month) in the same longitudinal series. 
Every two weeks, soft tissues and all teeth in the mouth were examined for possible side effects from the rinse such as ulcerations, lesions and tooth staining. Compliance check-off sheets were periodically examined to ensure adherence of protocol.

Student's t-test, Multivariate ANOVA and Exact F statistics were performed on $\triangle \mathrm{F}, \mathrm{Q}$ and the lesion size values to determine whether there were significant differences between the two groups before and after the treatment regimens. A significance level of $p<0.05$ was used in all statistical tests.

\section{Results}

The fluorescent images captured by the QLF device are shown in Figure 2. Keeping the light source perpendicular to the facial surface of the tooth resulted in reproducible images. The Inspektor Pro software enabled the use of contour points, which automatically compensated for the changes in distance between the camera and the lesion during imaging as shown in Figures 2 (A, B, C and D). In total, 12 subjects were originally included in this pilot trial ( 8 male and 4 female) but the experimental group had lost two subjects who withdrew voluntarily from the trial. The descriptive statistics of variables at different time points are depicted in Table 1. There was no significant difference between the baselines and at the end of the three-month rinsing period for $\Delta \mathrm{F}, \Delta \mathrm{Q}$, and for changes in the WSA ( $>>0.05)$. Both the experimental and the control rinse group showed no inhibition of demineralization nor remineralization towards the end of the third month when compared to the beginning of the trial. Multivariate ANOVA test criteria and exact $\mathrm{F}$ statistics for the hypothesis of no
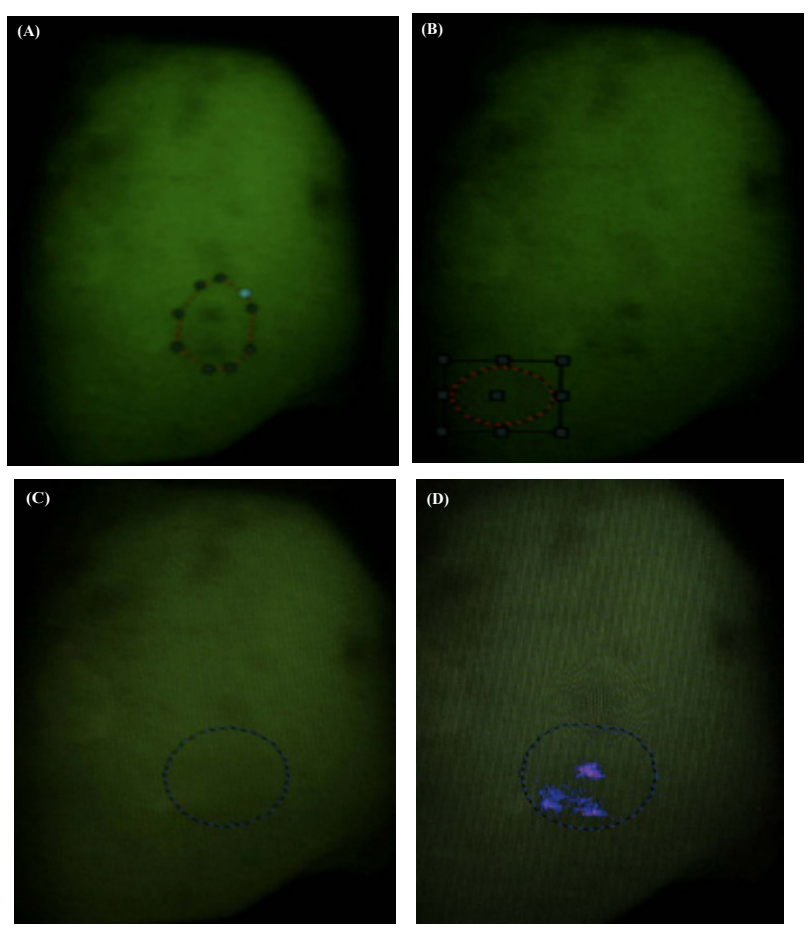

Figure 2: The captured images of the tooth were quantitatively analyzed with the QLF software. 1A depicts the selected lesion of interest. 1B the software selects sound area of the tooth structure not affected by caries and simulates sound enamel in the actual area of demineralization by using fluorescent radiance along the line of the lesion shown in 1C. 1D depicts the loss of fluorescent intensity in the lesion calculated by the difference in fluorescent radiance between the simulated sound area and the actual carious area and is expressed as a percentage of that of sound enamel.

\begin{tabular}{|c|c|c|c|c|c|c|c|}
\hline \multirow{2}{*}{ VARIABLE } & \multicolumn{3}{|c|}{ Control } & \multicolumn{3}{|c|}{ Experimental } & \multirow{2}{*}{$\begin{array}{c}\text { t-test } \\
\mathrm{p} \text {-value }\end{array}$} \\
\hline & $\mathbf{N}$ & MEAN & SD & $\mathbf{N}$ & MEAN & SD & \\
\hline$\Delta \mathrm{F} 0$ & 6 & -9.7433 & 3.0134 & 6 & -8.7517 & 1.4313 & 0.4897 \\
\hline$\Delta \mathrm{F} 1$ & 6 & -10.7833 & 1.6185 & 6 & -9.0617 & 2.7074 & 0.4669 \\
\hline$\Delta \mathrm{F} 2$ & 6 & -10.0633 & 1.6185 & 5 & -10.4780 & 2.5261 & 0.7612 \\
\hline$\Delta \mathrm{F} 3$ & 6 & -9.5317 & 2.5867 & 4 & -8.2575 & 1.0257 & 0.3138 \\
\hline$\Delta \mathrm{Q} 0$ & 6 & -12.5317 & 9.6694 & 6 & -7.1600 & 5.5389 & 0.3110 \\
\hline$\Delta \mathrm{Q} 1$ & 6 & -17.9667 & 4.6736 & 6 & -24.1300 & 29.9636 & 0.6388 \\
\hline$\Delta \mathrm{Q} 2$ & 6 & -23.8467 & 19.8083 & 5 & --29.9900 & 29.1321 & 0.7009 \\
\hline$\Delta Q 3$ & 6 & -10.7050 & 9.3164 & 4 & -12.5700 & 13.4974 & 0.8195 \\
\hline WSAO & 6 & 1.1933 & 0.8483 & 6 & 0.8433 & 0.6101 & 0.4330 \\
\hline WSA1 & 6 & 1.6800 & 0.2388 & 6 & 1.0767 & 1.7450 & 0.4384 \\
\hline WSA2 & 6 & 2.3483 & 1.8155 & 5 & 2.5080 & 2.3706 & 0.9050 \\
\hline WSA3 & 6 & 0.9733 & 0.7183 & 4 & 1.4900 & 1.6670 & 0.5920 \\
\hline
\end{tabular}

overall group effect also showed no significant difference between the two treatment groups with the p values being 0.7514 . No side effects from the rinses were observed.

\section{Discussion}

$\mathrm{MCH}$ is a long-term care facility for patients with chronic and complex health problems. This pilot trial employed randomized double blind trial with subjects with differing caries risks and prevalence. The present study is among the first to report the use of QLF as a cariesmonitoring device in this high-risk for caries progression group. The results of lesion not progressing further when rinsed with either calcium or a fluoride rinse are encouraging. Rinsing is a simple procedure and requires minimum cooperation even among patients with complex and chronic health problems as seen in this study. In this study the extent of remineralization produced by the experimental rinse was comparable to the control $\mathrm{NaF}$ rinse. Based on the results, it would seem ideal if the fluorescence loss had been monitored just before the subjects started the rinsing protocol as this would have given the actual fluorescence loss and the actual white spot size just before the trial began. This limitation would be taken into consideration for the planned larger clinical trial with the same rinses for a year. We presume that if the rinsing had progressed to at least 6 months there would have been comparable changes in the mineral content before and after the clinical trial. Also two subjects withdrew voluntarily and the small population size (apart from not being the ideal age group) may have affected the study outcome.

Calibration of an examiner before embarking upon clinical trials is vital for the standardization of any clinical trials. Trained professionals from Inspektor Research Systems calibrated the principal investigator both in the image acquisition and the analysis before the start of this trial. In this study we have demonstrated the capability of QLF to monitor changes in fluorescence due to changes in mineral content. Based on our results, QLF can be used as a caries monitoring tool to use in a dental clinic to screen the remineralization potential of any anticaries reparative agents that are formulated to enhance remineralization or inhibit demineralization in an caries active individual. One of the problems facing the dental practioner is how to determine the risk of bacterial challenge in a timely fashion [14]. The fact that QLF monitored small changes in mineral content over the duration of three months implies the reliability of QLF in caries risk assessment. This would enable practicing dentist to intercede the spread of caries in a caries active individual with fluoride or other remineralization therapies. Literature is abundant with scientific evidence pertaining to the validity, reproducibility and sensitivity of QLF technique with traditional methods like chemical analysis, transverse micro radiography and longitudinal micro radiography for detection and quantification of caries lesion $[10,13,19,20]$. Also QLF has proven 
to detect demineralized surface 9.5 times more than the traditional clinical examination [21]. QLF as a caries-monitoring device was successfully implemented in our pilot clinical trial based on the optical principles. Tooth is a natural fluorescing medium with light absorption and scattering properties. QLF utilizes the fluorescence properties of the tooth and distinguishes between caries and surrounding sound enamel [10]. Accordingly, if a portion of the tooth is affected by caries or demineralization then the amount of fluorescence emitted by this particular spot may be limited than from the surrounding sound tooth structure. When the QLF device captures this image, the lesion appears as a dark spot surrounded by favorably luminescent sound enamel as shown in Figure 1A. The caries detection was easier and devoid of any reflections on the captured images due to the optimization of blue and yellow filters combination as shown in Figures 1 (A and B). Also, the subjects were very impressed on seeing their own teeth quantitatively assessed on the computer screen and were motivated to do more thorough rinsing hoping to see a decrease in the size of the white spot lesion. Based on this we presume that this would be an added benefit of QLF over traditional caries diagnosis. Moreover, the ability of the software to accurately set and guide the investigator to capture images of a lesion in the same co-ordinates as the initial lesion images argues favorably to the reliability of the device. In order to maintain a reproducible hydration state before images were captured by QLF the subjects were asked to swipe the teeth with their saliva. This ensured that minimal hydration would not affect the fluorescence radiance or the optical properties of the tooth under investigation. It should also be noted that only one anterior tooth was selected as a master lesion. It ranged from the central incisors to mandibular canines with varying thicknesses in the subjects. However, previous studies have shown that neither thickness nor the bucco-lingual dimension of the tooth influences the fluorescence radiance of the tooth as long as a dentine layer is present [22]. The limitation that is worth investigating further is the fact that QLF readings may be hindered by the presence of plaque as shown in Figure 3. Image analysis of lesions superimposed with the plaque areas may skew up the data by acting as confounding variables. To overcome this limitation, prophylactic cleaning of the teeth prior to QLF readings can ease the effectiveness of this device. Also, the presence of stains may give an impression of an incipient caries when using the QLF device because it appears dark. However in our study when analyzing the stained lesion over a period of time there appeared to be no improvement in the mineral quantity, since process of caries

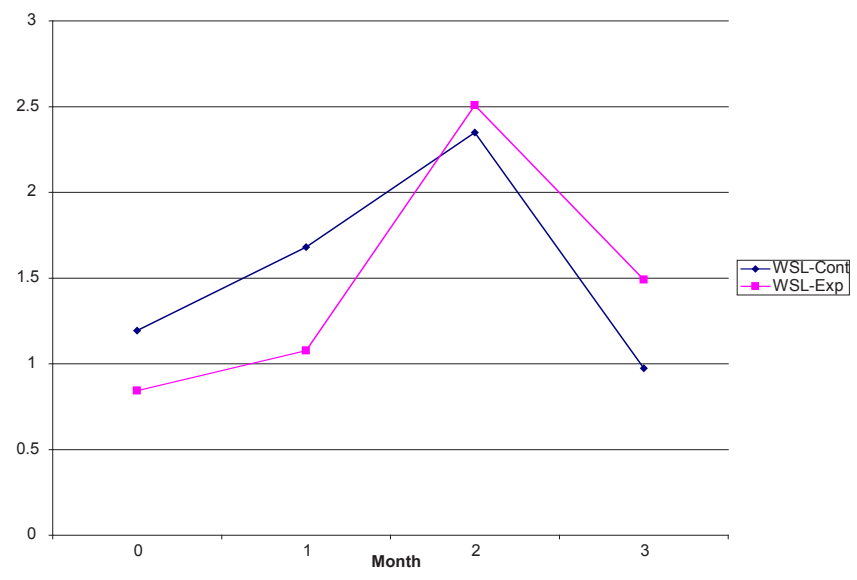

Figure 3: Changes in white spot lesion size for both the experimental and the control group indicated no remineralization at the end of the three-month period. The inserted image depicts no change in size of the lesion before and after the trial.

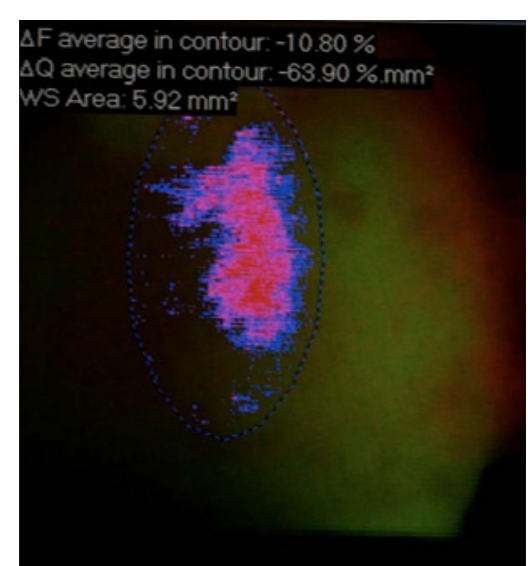

Figure 4: QLF reading may be skewed by the presence of plaque captured along the periphery and cervical areas of the tooth.

is dynamic, this makes the QLF device appropriate in monitoring any preventive regimen therapies on caries. Furthermore, it would be interesting to investigate if the QLF device could be used a diagnostic tool to monitor the efficiency of any vital bleaching protocol based on fluorescence properties.

This pilot clinical trial measured small changes in mineral content of white spot or early enamel lesions monitored by the QLF device and based on the results of this three-month trial the null hypothesis was accepted. However, we need to design a larger trial including more subjects for at least six-month duration. Nevertheless, QLF device seems appropriate to longitudinally monitor mineral changes over time and would complement any clinician's remineralization therapy in a caries active individual. We speculate that this would be an important armamentarium in a dental office setting.

\section{Acknowledgements}

This clinical trial was supported by the Basil Bibby Research Fellowship grant from Eastman Dental Center at University of Rochester. We would like to thank Mr. Joe Moore (Nurse Manager, MCH, Rochester, NY), Ms. Pat Lavigne (Registered Nurse, MCH, Rochester, NY), Dr. Gene Watson (Eastman Dental Center, Rochester, NY), Dr. John Featherstone (University of California at San Francisco) Dr. Dominic Zero (Indiana University), and Dr. Larry Chow are the American Dental Association Health Foundation (Gaithersburg, MD) for their scholarly input and technical support in this pilot clinical trial. We are indebted to Mr. Steve Prudue and to Inspektor Dental Care (Netherlands) for letting we borrow the QLF diagnostic system.

\section{References}

1. Horowitz AM (2004) A Report on the NIH Consensus Development Conference on Diagnosis and Management of Dental Caries Throughout Life. J Dent Res 83: 15-17.

2. Brown LJ, Winn DM, White BA (1996) Dental caries, restoration and tooth conditions in US adults, 1988-1991. Selected findings from the Third National Health and Nutrition Examination Survey. J Am Dent Assoc 127: 1315-1325.

3. Angmar-Mansson B, ten Bosch JJ (2001) Quantitative light-induced fluorescence (QLF): a method for assessment of incipient caries lesions. Dentomaxillofac Radiol 30: 298-307.

4. Heinrich-Weltzien R, Kuhnisch J, van der Veen M, de Josselin de Jong $E$ Stosser L (2003) Quantitative light-induced fluorescence (QLF)--a potential method for the dental practitioner. Quintessence Int 34: 181-188.

5. Pretty IA, Hall AF, Smith PW, Edgar WM, Higham SM (2002) The intra-and inter-examiner reliability of quantitative light-induced fluorescence (QLF) analyses. British Dental Journal 193: 105-109.

6. Angmar-Maansson B, Ten Bosch JJ (1987) Optical Methods for the Detection and Quantification of Caries. Advances in Dental Research 1: 14-20. 
Citation: Vaderhobli R, Saunders L, Billings R, Feng C, Malmstrom H (2012) Clinically Assessing Remineralizing Therapies using Quantitative Lightinduced Fluorescence: A Pilot Study. Dentistry 2:112. doi:10.4172/2161-1122.1000112

7. Angmar-Mansson B, ten Bosch JJ (1993) Advances in methods for diagnosing coronal caries--a review. Advances in Dental Research 7: 70-79.

8. Ten Bosch JJ, Angmar-Mansson B (1991) A review of quantitative methods for studies of mineral content of intra-oral caries lesions. J Dent Res 70: 2-14.

9. de Josselin de Jong E, Sundstrom F, Westerling H, Tranaeus S, et al. (1995) A new method for in vivo quantification of changes in initial enamel caries with laser fluorescence. Caries Res 29: 2-7.

10. van der Veen $\mathrm{MH}$, de Josselin de Jong E (2000) Application of quantitative light-induced fluorescence for assessing early caries lesions. Monogr Oral Sci 17: 144-162.

11. Shi XQ. Tranaeus S, Angmar-Månsson B (2001) Comparison of QLF and DIAGNOdent for quantification of smooth surface caries. Caries Res 35: 21-26.

12. Ando M, Hall AF, Eckert GJ, Schemehorn BR, Analoui M, et al. (1997) Relative ability of laser fluorescence techniques to quantitate early mineral loss in vitro. Caries Res 31: 125-131.

13. al-Khateeb S, ten Cate JM, Angmar-Mansson B, de Josselin de Jong E, Sundstrom G, et al. (1997) Quantification of formation and remineralization of artificial enamel lesions with a new portable fluorescence device. Advances in Dental Research 11: 502-506.

14. Featherstone JD (2000) The science and practice of caries prevention. The Journal of the American Dental Association 131: 887-899.

15. Bader JD, Shugars DA, Bonito AJ (2001) A systematic review of selected caries prevention and management methods. Community Dent Oral Epidemiol 29: $399-411$.
16. Zero DT (2006) Dentifrices, mouthwashes, and remineralization/caries arrestment strategies. BMC Oral Health 6: s9.

17. Hohmann AA, Parron DL (1996) How the new NIH guidelines on inclusion of women and minorities apply: Efficacy trials, effectiveness trials, and validity. Journal of Consulting and Clinical Psychology 64: 851-855.

18. Ando M, van der Veen MH, Schemehorn BR, Stookey GK (2001) Comparative Study to Quantify Demineralized Enamel in Deciduous and Permanent Teeth Using Laser-and Light-Induced Fluorescence Techniques. Caries Research 35: $464-470$.

19. Lagerweij MD, van der Veen MH, Ando M, Lukantsova L, Stookey GK (1999) The Validity and Repeatability of Three Light-Induced Fluorescence Systems: An in vitro Study. Caries Research 33: 220-226.

20. Hafstrom-Bjorkman U, Sundstrom F, de Josselin de Jong E, Oliveby $A$, Angmar-Mansson B (1992) Comparison of laser fluorescence and longitudinal microradiography for quantitative assessment of in vitro enamel caries. Caries Res 26: 241-247.

21. Ferreira Zandoná AG, Isaacs RL, van der Veen M, Eckert GJ, Stookey GK (1998) Comparison between light-induced fluorescence and clinical examinations for caries detection (abstract 86). Caries Res 32: 96.

22. Amaechi BT, Higham SM (2002) Quantitative light-induced fluorescence: A potential tool for general dental assessment. Journal of Biomedical Optics 7: 7-13. 